\title{
COMPTE-RENDUS DE CONGRES ET REVUE DE LA LITTERATURE INTERNATIONALE
}

avec la collaboration de :

L. BUJAN

J.C. CZYBA

M.A. DROSDOWSKY

J.F. GUERIN

S. HAMAMAH

H. LEJEUNE

K. OSTROWSKI

S. ZERAH 


\section{Compte-rendus de Congrès et Revue de la littérature internationale}

\section{COMPTE-RENDUS DE CONGRES}

$269 \quad$ L'Andrologie dans les années quatre vingt dix.

S. ZERAH

3ème Symposium à Norfolk (Jones Institute for Women’s Health), 13-14 mars 1997.

274 Le désir d'enfant chez les couples VIH sérodifférents.

J.F. GUERIN, L. BUJAN

1ères journées nationales, Toulouse, 21-22 mars 1997.

\section{ENDOCRINOLOGIE}

277 Modulation par les androgènes de l'ARN messager des récepteurs de l'acide rétinoïque dans la prostate, les vésicules séminales et le rein, chez le rat.

H.F.S. HUANG, M.T. LI, S. VON HAGEN ET AL.

Endocrinology, 138, 2, 553-559, 1997.

278 La cycline D2 est un gène répondant à la FSH et impliqué dans la prolifération cellulaire et l'oncogénèse gonadique.

P. SiCINSKI, J.L. DONAMER, Y. GENG ET AL.

Nature, $384: 470-474,1996$.

\section{ASSISTANCE MEDICALE A LA PROCREATION}

280 Index de difformité spermatique : prédiction fiable de la fécondation in vitro. N. AZIZ I. BUCHAN, C. TAYLOR ET AL.

Fertility-Sterility, 66 : 1000-1008, 1996.

281 Modalités de récupération de la fertilité après vasectomie : analyse du coût et de l'efficacité.

C.P. PAVLOVICH, P.N. SCHLEGEL

Fertility-Sterility, 67 : 133-141, 1997. 


\section{COMPTE-RENDUS DE CONGRES}

\section{L'Andrologie dans les années quatre vingt dix (Andrology in the Nineties)}

\section{S. ZERAH}

Compte-rendu du 3ème Symposium qui a eu lieu à Norfolk (Virginie-USA) les 13 et 14 Mars 1997 au Jones Institute for Women's Health

Le premier jour a été consacré à la biologie du sperme et aux différents tests biologiques dans le domaine de l'andrologie.

Le deuxième jour a été consacré aux approches thérapeutiques de l'infertilité masculine.

\section{Biologie de la fécondation Ryuzo YANAGIMACHI}

Malgré les progrès considérables réalisés ces dix dernières années, plusieurs questions ne sont pas encore élucidées :

- Comment un spermatozoïde fécondant arrive à l'endroit de la fécondation?

- Où et quand un spermatozoïde est capacité avant la fécondation?

Il semble que certains composants du liquide folliculaire libérés dans l'oviduc pendant l'ovulation attireraient le spermatozoïde humain de la partie inférieure du tractus génital féminin.

Le passage du spermatozoïde à travers le cumulus et la ZP (Zone Pellucide) nécessite des conditions physiques (vigoureuse mobilité) et biochimiques $=$ présence de Progestérone dans le cumulus (la fécondation est meilleure en présence de cumulus) et libération de calcium intracellulaire.

Des changements physiologiques qui surviennent dans la membrane au niveau du segment équatorial de l'acromose, concomittants de la réaction acrosomique rendent la membrane du spermatozoïde fusiogène et importance du centrosome (essentiel pour la fécondation chez l'homme).

La nature de ces changements n'est pas complètement connue. C'est la fusion des membranes des gamètes qui provoque l'activation de l'ovocyte.
Intéraction spermatozoïde-Zone Pellucide Patricia SALING

L'interaction initiale entre le spermatozoïde et l'ovocyte survient au niveau de la zone pellucide : c'est la ZP3 (glycoprotéine de la zone pellucide) qui permet à la tête du spermatozoïde de s'attacher, puis intervient la ZP2.

L'étude de l'activation des récepteurs a été possible grâce à la biologie moléculaire (la tyrosine phosphorylation est ZP3 dépendant).

Quand le spermatozoïde s'attache à la ZP, la réaction acrosomique se produit très rapidement.

\section{Réaction acrosomique}

Klaus-Dieter HINSCH, E. HINSCH

La ZP3 joue un rôle essentiel pendant la fécondation. Elle fonctionne comme un récepteur spécifique pour le spermatozoïde et induit la réaction acrosomique.

La conversion de la ZP3 empêche la polyspermie ("zona hardening"). Si on ajoute de l'anti ZP3 pour effectuer l'Hémizona-test, le résultat baisse de façon significative.

Un autre facteur actif sur la réaction acrosomique est la Progestérone. La Progestérone stimule l'entrée du Ca++ dans le spermatozoïde.

\section{Facteurs agissants sur la fonction spermatique John AITKEN}

Le spermatozoïde humain est une cellule très différenciée aux nombreuses propriétés dont l'objectif est de féconder l'ovocyte.

Les facteurs qui peuvent affecter les fonctions spermatiques sont aussi bien les régulateurs physiologiques de la capacitation et de la réaction acrosomique que les facteurs pathologiques responsables de la diminution du potentiel de fécondation dans l'infertilité masculine.

Les régulateurs de la capacitation (capacité de recevoir les signaux du complexe cumulus-ovocyte) sont la présence de bicarbonate extracellulaire, calcium et protéines et la génération de cAMP et de peroxyde d'hydrogène.

La présence de ces facteurs augmente les taux de tyrosine phosphorylation, augmente la fluidité membranaire (par des mécanismes qui impliquent la stimulation de la tyrosine kinase, inhibition de la tyrosine phosphatase et le déplacement du cholestérol de la membrane plasmique). 
L'induction de la tyrosine phosphorylation est un élément important de la capacitation = la cellule peut répondre au flux calcique induit par la Progestérone et quand le spermatozoïde touche la surface de l'oeuf (ZP3) provoquer la réaction acrosomique.

\section{Le chromosome $\mathbf{Y}$ et l'infertilité masculine David C. Page}

C'est dans leur laboratoire, à Cambridge, en 1992, qu'ont été établies les premières cartes intelligibles du chromosome $\mathrm{Y}$ humain. Ceci a permis d'explorer les hypothèses selon lesquelles certaines infertilités masculines pourraient être dues à des anomalies du chromosome Y. Il a été trouvé une délétion de novo d'une certaine partie du chromosome $\mathrm{Y}$ dans $13 \%$ d'hommes avec une azospermie non obstructive et chez un plus petit nombre d'hommes avec une oligospermie sévère (pas d'anomalies de chromosomes Y chez le père).

Ces délétions concernent une région où devait être trouvé un ou plusieurs gènes responsables de la spermatogenèse (un facteur de l'azospermie AZF).

Ces hommes sont, par ailleurs, en bonne santé suggérant que l'AZF est un locus responsable de la stérilité. En l'absence d'AZF, la production de spermatozoïdes est diminuée ou inexistante et dans les cas les plus sévères, les testicules ne contiennent pas de cellules souches.

Il semblerait que l'AZF facilite la différenciation des cellules primordiales en spermatogonies ou influence la destinée des cellules souches qui, chez l'homme normal, ont 3 alternatives d'évolution:

- prolifération ;

- dégénérescence ;

- différenciation.

Ces délétions concernent des gènes étroitement liés = DAZ (Deleted in Azospermia) exprimé spécifiquement dans les spermatogonies et leurs descendants, les spermatocytes primaires $=\mathrm{il}$ semble coder pour une RNA binding protéine.

L'absence de DAZ serait la cause des défauts sévères de la spermatogenèse (ou au moins $\mathrm{y}$ contribuerait) chez les hommes où l'on observe une délétion du gène.

\section{Facteurs prédictifs en fécondation}

Les 3 facteurs les plus importants sont:
- la morphologie des spermatozoïdes ;

- la mobilité ;

- l'intéraction ovocyte spermatozoide.

1) Thinus KRUGER a insisté une fois de plus sur la valeur prédictive évaluée par "strict criteria" de la morphologie des spermatozoïdes en AMP.

Il semble qu'il y aurait actuellement un consensus :

Thinus KRUGER a analysé 216 articles (entre 1980 et 1996).

Si le \% de spermatozoïdes normaux est $<5 \%$,

le taux de fécondation est de $30,3 \%$

le taux de grossesse de $6,79 \%$

$\mathrm{si}>5 \%$,

taux de fécondation $76,89 \%$

taux de grossesse $20,92 \%$.

2) Mary C. MAHONY a présenté l'analyse informatisée des mouvements des spermatozoïdes.

Une expérimentation sur des spermatozoïdes de singe a permis d'étudier l'intérêt de l'hyperactivation (AMP cyclique dépendant) pour la fécondation et de définir les paramètres importants :

VSL : vitesse de progression de la cellule

ALH : amplitude latérale de la tête

VCL : vitesse curvi linéaire

\section{3) Intéraction ovocyte spermatozoïde Daniel FRANKEN}

La capacité d'une population de spermatozoïdes de s'attacher à la zone pellucide de l'ovocyte est un facteur crucial dans la fécondation chez les mammifères. Pour l'évaluer, nous disposons d'un test "Hemizona-assay" qui a l'avantage d'être un test fonctionnel comparant des populations de spermatozoïdes fécondants et non fécondants dans le même essai. Le facteur limitant de cet esssai est la possiblité de disposer d'ovocytes en métaphase II (même non fécondés).

\section{Intérêt du contrôle de qualité dans un laboratoire d'andrologie Christopher DE JONGE}

Le contrôle de qualité est déjà bien connu des laboratoires de biologie médicale et des grandes entreprises. 
Actuellement, on assite à un regroupement dans les laboratoires d'andrologie, de "nouveauvenus" venant de différents horizons qui doivent intégrer et appliquer les notions de qualité.

Le contrôle des instruments, l'évaluation des variations est un point important. Mais, le principe de contrôle de qualité relève d'une plus large philosophie. Le directeur du laboratoire qui est un "manager" doit développer une mission et une vision de la qualité. C'est le TGM (Total Qualité Management) qui est complété par le CGI (Continuous Quality Improvement). C'est une approche systématique et continue de l'organisation pour améliorer tous les procédés qui permettent de fournir des services et des produits de qualité.

Les acteurs concernés sont :

- le patient,

- l'andrologue (ou tout autre autre spécialiste dans d'autres domaines);

- le personnel.

On n'atteint la qualité que par l'intermédiaire des personnes et de l'éducation. Il faut mettre en place des procédures, des fiches quotidiennes de contrôle pour repérer toutes variations et des fiches de satisfaction des clients.

Enfin, se souvenir de la phrase d'Einstein : "L'imagination est plus importante que la connaissance".

Johannes LH EVEN a souligné l'importance des études et des travaux de recherches très rigoureux ayant recours aux statistiques. L'andrologie (comparée à d'autres disciplines) commence seulement à accepter le besoin d'avoir des essais contrôlés et randomisés (exemple de la varicocèle).

\section{Conduite clinique à tenir devant une infer- tilité masculine}

Sergio OEHNINGER

Après avoir établi l'historique et l'examen médical d'un patient, ainsi que les examens urologiques et endocrinologiques et des examens répétés du sperme, il faut rechercher si les causes sont pré-testiculaire, testiculaire ou posttesticulaire.

Il faut identifier les anomalies morphologiques ou fonctionnelles. Etudier les anomalies de migration, de mobilité, la réaction acrosomique, l'attachement à la zone pellucide et la pénétration, la décondensation.

Certaines caractéristiques des spermatozoïdes peuvent conduire à révéler certains désordres cellulaires, comme l'immaturité (associée à des modifications de certaines marqueurs biochimiques) ou la nécrose.

On peut identifier des anomalies moléculaires (structures membranaires, récepteurs) ou chromosomiques et génétiques.

On peut traiter certains cas par la chirurgie ou des traitements hormonaux. Mais, dans la plus grande majorité des cas, on fait appel aux techniques d'AMF, surtout depuis l'ICSI.

Des grossesses conduisant à la naissance d'enfants bien portants ont été obtenues dans de nombreux cas d'infertilité masculine.

Les efforts doivent maintenant porter sur la compréhension des mécanismes de dysfonctionnement : défauts de la spermatogénèse, maturation, interaction avec les composants féminins, signaux et échanges avec l'ovocyte.

\section{Statégies médicales pour le traitement de l'infertilité masculine}

John AITKEN

L'étiologie de plus de $90 \%$ des cas d'infertilité masculine étant inexpliquée, les opportunités de traitements médicaux appropriés sont très limitées.

Contrairement aux stérilités féminines, les causes endocrines sont rares :

- hypogonadisme, hypogonadotrophique congénital (Kallmann's syndrome) ou acquis (conséquence de tumeurs) ou de maladies inflammatoires.

Dans ces cas, l'apport exogène de gonadotrophines semble donner de meilleurs résultats dans les lésions acquises.

L'administration empirique de traitements hormonaux n'a aucune valeur thérapeutique (antioestrogènes, androgènes, gonadotrophines, bromocriptine) à des patients sans diagnostic de désordre.

En cas de suspicion d'infection, les traitements antibiotiques ne donnent pas toujours les résultats attendus. En effet, la présence de leucocytes 
n'est pas toujours un signe d'infection, les antibiotiques utilisés ne pénétrent pas toujours au site de l'infection. Une conséquence importante de la présence de leucocytes est la peroxydation. Les spermatozoïdes sont très vulnérables ; dans le sperme, ils sont protégés par le plasma séminal (présence de catalase libre, Hypotaurine, zinc). Si on lave, il faut ajouter dans les milieux des antioxydants (catalase, Hypotaurine). Le Percoll est la meilleure technique de préparation (séparation des spermatozoïdes des leucocytes et autres débris), l'immaturité des gamètes, la présence de restes cytoplasmiques dans la pièce intermédiaire peuvent également être la source de radicaux libres et être la cause de la baisse de la fertilité.

On peut administrer des antioxydants comme la Vitamine E (Tocoferol $300 \mathrm{mg} /$ jour).

\section{Traitement chirurgical de l'infertilité masculine Marc GOLDSTEIN}

Les progrès techniques de la microchirurgie ont fait considérablement progresser la chirurgie de l'infertilité masculine.

En ce qui concerne les varicocèles :

- Il est très important de bien sélectionner l'indication et de classer le varicocèle. On trouve un varicocèle dans $15 \%$ de la population générale, dans $35 \%$ de cas d'infertilité primaire, dans $81 \%$ en cas d'infertilité secondaire. L'index de fertilité augmente après intervention surtout pour les grades III ;

- Les résultats dépendent de la technique opératoire utilisée. Il est fondamental de préserver les artères (Marc GOLDSTEIN a montré une vidéo cassette très détaillée $=$ intervention en 30 minutes en ambulatoire).

Pour les ponctions épididymaires et les biopsies testiculaires suivies d'ICSI, la moyenne des taux de grossesse est de $50 \%$. M. GOLDSTEIN conseille d'attendre 6 mois entre 2 biopsies testiculaires. Si on fait une opération reconstructrice, on recueille le sperme épididymaire pour une éventuelle ICSI ultérieure avec le sperme congelé.

\section{Rôle de l'insémination intrautérine dans l'approche thérapeutique de l'infertilité masculine Willem OMBELET}

De nombreuses variables peuvent influencer les résultats :
- La préparation des patientes : les cycles stimulés donnent de meilleurs résultats que les cycles spontanés. Mais, il faut une surveillance attentive par échographie, et doser l'oestradiol pour éviter les grossesses multiples.

- L'insémination intra-utérine est supérieure à l'insémination intracervicale ;

- Le déclenchement se fait par 5000 ou 10000 UI HCG.

On obtiendrait de meilleurs résultats en faisant des inséminations successives : une à $14 \mathrm{~h}-18 \mathrm{~h}$, une à $34 \mathrm{~h}-36 \mathrm{~h}$ après déclenchement (ceci à été démontré par une étude randomisée, d'une part, parce que le moment de l'ovulation n'est pas toujours bien synchronisé, d'autre part, parce que le deuxième échantillon de sperme est souvent meilleur que le premier).

- On obtient de bons résultats après un premier cycle, on conseille trois cycles. A partir du quatrième cycle, les résultats sont beaucoup moins bons, il faut passer à une autre technique d'AMP.

- En cas de problèmes immunologiques, les IIU donnent de bons résultats.

Conclusion : L'IIU peut être envisagée en première intention avant toute autre technique d'AMP si on obtient au moins 1 million de spermatozoïdes mobiles après préparation.

\section{Résultats de l'ICSI \\ André VAN STEIRTEGHEM}

Depuis 1991 (le premier bébé est né en janvier 1992), l'ICSI apporte une solution à des couples souffrant d'infertilité depuis longtemps et qui ne pouvaient pas bénéficier d'une technique d'AMP à cause de la faible quantité de spermatozoïdes mobiles dans le sperme.

L'ICSI peut être également appliquée à des spermatozoïdes provenant de ponctions épididymaires ou de biopsies testiculaires. Cette technique est également utilisée pour le sperme conservé avant traitement anticancéreux.

$70 \%$ des ovocytes en Méta II sont fécondés, les $2 / 3$ des ovocytes à $2 \mathrm{PN}$ donnent des embryons qui sont transférés ou congelés. On obtient $32 \%$ de grossesses, $24 \%$ évolueront vers la naissance d'un bébé.

Cette technique nécessite : 
- un excellent équipement ;

- une grande expérience ;

- une grande rigueur dans la technique, par exemple, il faut respecter le temps de contact avec la Hyaluonidase (pour enlever le cumulus) sinon on obtient une parthénogenèse.

Dans son exposé sur ses recherches actuelles, Jacques COHEN a insisté sur l'importance de la position de l'ovocyte et du globule polaire, et de l'endroit où on dépose le spermatozoïde (pour avoir la meilleure qualité embryonnaire, il faut le déposer à la position 8 heures).

On n'obtient pas de fécondation avec des spermatozoïdes à tête ronde et s'il n'y a que des spermatozoïdes immobiles. Par contre, YANAGIMACHI a montré que des spermatozoïdes à morphologie anormale n'ont pas obligatoirement un génome anormal.

Il semble qu'avec les spermatozoïdes congelés après ponction épididymaire, les taux d'implantation et de grossesses évolutives sont moins bons.

L'équipe de Bruxelles fait un caryotype spermatique chez l'homme avant ICSI.

Le suivi des enfants est très important = sur 1474 enfants nés, on ne montre pas d'augmentation de malformations. On note 36 malformations congénitales majeures soit $2,4 \%$, ce qui correspond à la moyenne des naissances dans la population générale.

\section{ICSI avec spermatides}

Martine NIJS, P. VANDERZWALMEN

Les travaux d'YANAGIMACHI (présentés par ailleurs à ce symposium) ont montré qu'il y a la possibilité de faire une ICSI avec cellules spermatiques immatures (spermatides) sur l'animal en particulier chez la souris (étude sur 5 générations de souris avec ICSI avec spermatides).

Chez l'homme, après l'obtention de quelques grossesses (Fischel \& All, Vanderzwalmen and all 1995) et deux naissances à partir de spermatides recueillis dans l'éjaculat (Tesarik), l'équipe a entrepris une étude chez des patients azoospermiques (cette technique doit encore être considérée dans une phase de recherche) $=29$ patients ont été inclus dans cette étude : 5 grossesses ont été obtenues sur 39 transferts $=1$ avortement, 1 grossesse en cours, 3 naissances (dont 2 après injection de spermatides allongés et matures). La qualité des embryons obtenus est moins bonne qu'en FIV.

Le taux d'implantation est très faible $(5,5 \%)$.

Il est très important de faire, au préalable, une biopsie diagnostic $=$ si on a obtenu des spermatozoïdes et qu'on n'en trouve plus, on peut essayer d'injecter une spermatide, si on n'a pas vu de spermatozoïdes, les résultats sont nuls.

La taille de la spermatide injectée doit être inférieure à $8 \mathrm{~mm}$ (taille d'un érythrocyte). L'injection doit se faire pas plus d'une demi heure après le recueil, sinon dégénérescence.

Il ne faut pas oublier le risque de transmission de délétions du chromosome $Y$ ou d'autres anomalies. Il est indispensable, quand on a recours à ces techniques, d'avoir une étude complète du dossier et du suivi de l'enfant né.

\section{La technique de micromanipulation en pratique clinique}

Suzan LANZENDORF

1) La technique de l'ICSI a été une révolution dans le traitement de l'infertilité masculine. Comme le nombre de centres effectuant cette technique ne cesse de croître, il est important de parfaitement comprendre tous les détails pratiques pour augmenter le taux de grossesses, en particulier dans les oligoteratospermies sévères et lorsqu'on recueille les spermatozoïdes à partir de biopsies testiculaires.

Par exemple :

- Si le sperme est très mauvais, on fait seulement deux lavages.

- Il faut faire très attention aux variations de température et d'atmosphère ;

- Il ne faut pas laisser les ovocytes plus de 10 secondes dans la hyaluronidase, pour enlever le cumulus. Le reste est enlevé à la pipette ;

- Ne pas laisser l'ovocyte plus de 15 minutes à l'extérieur de l'étuve, lorsqu'on injecte (on met + ou - d'ovocytes en fonction du temps qu'on met à injecter)

- L'immobilisation du spermatozoidde avant l'injection doit être agressive, il faut bien voir le courbure du flagelle avant d'injecter (surtout pour les spermatozoïdes épididymaires ou testiculaires). 
2) «Assited Hatching »

Cette technique s'applique dans les cas suivants :

- Age élevé de la patiente ;

- FSH élevée ;

- Zone pellucide épaisse ;

- Echecs après plusieurs tentatives.

Le trou dans la zone pellucide est fait avec du thyrode acide à ph 2,5 puis les embryons sont aussitôt lavés 6 fois avec du nouveau milieu et remis en milieu de culture.

3) Biopsie de blastomères

Ne peut s'effectuer que sur un embryon d'au moins 6 à 8 blastomères avec une pipette spéciale.

\section{Diagnostic pré-implantatoire William E. GIBBONS}

3 conditions :

- Le temps : il faut respecter la fenêtre d'implantation ;

- La sensibilité de la technique : la sensibilité pratique du diagnostic génétique demande une égale sensibilité théorique (habilité à amplifier la copie d'un seul gène à partir d'une seule cellule) ;

- La contamination : grâce à l'ICSI, on élimine toute contamination à partir des spermatozoïdes.

Une étude a été effectuée sur 323 patients ayant une absence congénitale des canaux déférents. On a recherché la mutation du gène de la mucoviscidose, 214 étaient porteurs (soit $65 \%$ ).

Facteurs influençant les taux de grossesses en AMP

\section{Suheil J. MUASHER}

L'infertilité est un problème de couple. De nombreuses variables interviennent pour obtenir une grossesse après AMP. Les facteurs importants sont:

- L'âge (dont dépend la qualité ovocytaire);

- L'âge ovarien déterminé par le taux basal de FSH, prédit la qualité de la stimulation ovarienne (pic d'E2 et nombre d'ovocytes recueillis) ;
- La politique de transfert :

- Nombre d'embryons replacés ;

- Jour du transfert : J2 ou J3 ;

- La qualité du cathéter ;

- La politique de congélation :

- sélection des embryons ;

- congélation des plus "beaux ";

- congélation à $2 \mathrm{PN}$, à $\mathrm{J} 2$ ou à J3 ;

- Les protocoles de stimulation : les gonadotrophines avec plus de FSH donnent des meilleurs résultats que celles avec un rapport égal FSH/LH.

- Les conditions de culture ,

- Assisted Hatching : une étude randomisée en double aveugle au Jones Institute sur des patients de 36 ans et plus n'a montré aucune différence ;

- Les nouveaux produits : FSH purifiée et FSH recombinante semblent devoir donner des résultats prometteurs :

- En cas d'hydrosalpinx : il faut opérer avant une FIV (à cause de l'embryotoxicité du fluide).

- Dosage des anticorps antiphospholipides au nombre de 6 pour Ig G et Ig M : si plus de 2 sont positifs, il faut traiter par l'aspirine ou l'héparine.

\section{Le désir d'enfants chez les couples VIH Sérodifférents}

J.F. GuERIN, L. BuJAN

Toulouse, 21-22 mars 1997

Quelle doit être l'attitude médicale face au désir d'enfant au sein d'un couple dont le partenaire est VIH+ ? Cette question ne doit se poser qu'après évaluation :

- de l'état général des 2 partenaires

- du projet parental au sein du couple

En théorie, une fécondation sera possible de 3 manières :

-> Sperme du partenaire inséminé au cours des rapports sexuels 
-> Sperme du partenaire traité en AMP (IAC, FIV ...)

$\rightarrow$ Sperme de donneur

\section{L'évaluation des risques de transmission du VIH}

Une étude multicentrique présentée par Mme VICENZI, a montré que sur 121 couples séro-différents qui n'ont pas utilisé les préservatifs de manière systématique, 12 séro-conversions ont été observées (soit un taux de 4,8 pour 100 personnes/années).

Plusieurs facteurs de risque ont été mis en évidence, en particulier la période de séro-conversion et l'existence d'infections génitales. Le taux de lymphocytes CD4 ainsi que la charge virale constituent des éléments pronostics valables.

\section{Le point de vue du clinicien (P. Massip)}

Une triple surveillance clinique, virale, et immunologique, doit être instaurée. Le succès actuel des trithérapies est indicutable, mais on peut craindre une adaptation progressive du virus à ces thérapeutiques. Le problème de la génotoxicité de celles-ci sur la lignée germinale, a été quasiment non évoquée jusqu'à présent, ce qu'on peut regretter.

\section{Le point de vue du virologue (C. Rouzioux)}

Le terme de "charge virale" regroupe 2 éléments : l'ARN viral, correspondant à des particules virales libres (mais beaucoup ne sont pas infectieuses), et l'ADN pro-viral, correspondant aux cellules infectées.

- L'ADN viral a été détecté chez $80 \%$ des sujets séro-positifs (Il faut faire attention aux fauxnégatifs, car le liquide séminal contient des inhibiteurs de P.C.R.). Il existe une bonne corrélation entre ARN viral dans le sperme et dans le sérum, néanmoins la charge sérique est toujours supérieure à la charge dans le sperme (d'un facteur 10 environ).

- La mise en évidence d'ADN pro-viral dans les cellules rondes (lymphocytes, macrophages) a montré que $50 \%$ de ces cellules étaient infectées. Une question très importante demeure d'actualité : le virus peut-il pénétrer dans le spermatozoïde, comme l'a suggéré une observation de BACETTI, ou simplement être adsorbé sur cette cellule ? Même dans cette dernière hypothèse plus "rassurante», le virus pourrait ainsi être véhiculé par le spermatozoïde jusqu'à l'ovocyte.

L'exposé a été conclu en précisant qu'aveç les techniques actuelles, on ne pouvait affirmer le risque 0 . Le seuil de détection correspond actuellement à 200 copies, mais est en abaissement constant grâce aux progrès de la technique.

\section{L'efficacité et les résultats de la prise en charge médicale}

- Les rapports sexuels «ciblés» (L. MANDELBROT)

Ici, l'intervention médicale se limite aux simples conseils, relatifs à la programmation des rapports sexuels afin d'optimaliser les chances de fécondation. Sur 104 grossesses suivies par l'équipe médicale entre 1986 et 1996, 68 ont été ainsi "programmées", 17 ayant été obtenues consécutivement à un rapport sexuel unique. Aucune séro-conversion n'a été observée dans les 3 mois suivant la conception, sur cette petite série. (Deux séro-conversions ont toutefois été observées au 7e mois de grossesse, en principe non liées aux rapports fécondants). Le risque de contamination apparait donc faible, mais difficilement chiffrable avec précision.

- L'insémination artificielle (IAC) avec sperme traité (A.E. SEMPRINI)

L'expérience italienne est unique au monde, au moins en ce qui concerne le grand nombre de cas traités : 1128 cycles d'IAC, correspondant à 354 femmes. 123 ont accouché (soit 17\% de grossesse/ cycle) et aucune contamination n'a été observée ni chez les femmes traitées, ni chez les 141 enfants nés. D'après les résultats de l'équipe italienne obtenus par P.C.R., on ne retrouve plus de charge virale à la fin du traitement du sperme (centrifugation sur gradient de Percoll, suivie de lavagemigration). Les « spermiologues » de l'auditoire ont toutefois été surpris par la chute brutale de la charge virale au cours de la dernière étape (lavage-migration), alors qu'elle restait élevée après le passage sur Percoll.

Ces données optimistes ont été tempérées par les résultats d'une étude expérimentale ( $S$. HAMAMAH) dans laquelle les auteurs ont infecté du sperme avec du VIH, puis l'ont centrifugé sur gradient de Percoll. Cette technique a permis de diminuer d'un facteur important (104) la charge virale, mais sans la faire disparaître totalement, même au bout de lavages répétés après passage sur Percoll. Les données sont 
donc en faveur d'une adsorbtion du VIH sur la membrane spermatique, voire d'une pénétration $\mathrm{du}$ virus dans la cellule, bien que les contaminations in vitro ne reflètent pas forcement la situation "naturelle".

- L'insémination artificielle avec sperme de donneur (IAD)

L'IAD représente la seule technique capable d'affirmer le risque 0 , en terme de contamination liée à l'acte médical, et en conformité avec la loi de Bioéthique (car il y a «risque de transmission à l'enfant d'une maladie d'une particulière gravitém). Le problème qui se pose est celui de la pratique d'une IAD chez un couple non stérile, qui devra donc faire le deuil d'une fécondité pourtant normale a priori. L'expérience la plus importante a été rapportée par le CECOS Cochin (P. JOUANNET et J.M. KUNSTMANN): parmi 185 couples qui ont consulté, 72 ont été effectivement pris en charge, 47 grossesses ont été initiées, et 34 enfants sont nés. Le taux de succès par cycle $(8 \%)$ était voisin de celui obtenu dans les indications conventionnelles d'TAD.

\section{En conclusion :}

Les risques inhérents à l'insémination par le sperme du conjoint, qu'elle soit naturelle (rapports sexuels programmés) ou artificielle (après traitement du sperme), paraissent faibles selon les données actuelles. Ils sont certainement inférieurs à $1 \%$ dans le cas des LAC, plus difficilement chiffrables pour les rapports sexuels "programmés“ ; de plus, ils dépendent de nombreux paramètres.

La responsabilité du médecin est bien sûr beaucoup plus engagée dans le cadre d'une Assistance Médicale à la Procréation que dans celui d'un simple conseil pour optimiser les chances de grossesses après rapports, même si beaucoup pensent que le médecin ne peut se décharger de toute responsabilité dans cette dernière situation, et que l'acte médical constitue une entité indivisible, qui va du conseil à l'acte technique.

L'intérêt présenté par la réalisation d'IAC après traitement du sperme réside dans la possibilité, avec l'amélioration prévisible de la sensibilité des techniques et de leur rapidité d'exécution, de tester la charge virale des spermatozoïdes traités avant insémination. Une bonne collaboration entre virologues et biologistes de la reproduction apparaît nécessaire.
Un certain nombre de règles devraient être respectées si un tel protocole était envisagé :

- l'accueil du couple au sein d'une équipe pluridisciplinaire, et évaluation de l'état général des deux partenaires ainsi que du projet parental ;

- la délivrance aux couples d'une information claire et détaillée des risques inhérents aux différentes méthodes de prise en charge ;

- l'obtention du consentement écrit des patients

- l'avis favorable d'une instance compétente (CCPPRB, CNMBR, etc)

- Si l'on ne veut courir aucun risque, la seule solution consiste en un recours au sperme de donneur, mais qui pose des problèmes d'autre nature, à la fois pour le couple (deuil de la paternité biologique), et pour l'institution représentée par le CECOS (aide à la conception d'un enfant qui risque d'être rapidement orphelin).

Quelle que soit la décision prise, il est souhaitable que celle-ci le soit après discussion du dossier au sein d'une équipe pluridisciplinaire, en concertation avec le couple. 


\section{ENDOCRINOLOGIE}

\section{Modulation par les androgènes de l'ARN messager des récepteurs de l'acide réti- noïque dans la prostate, les vésicules sémi- nales et le rein chez le rat.}

\section{Androgen modulation of the messenger ribonucleic acid of retinoic acid receptors in the prostate, seminal vesicles, and kidney in the rat.}

H.F.S. HUANG, M.-T. LI*, S. VON HAGEN**, Y.F. ZHANG***, R.J. IRWIN

Department of Surgery Section of Urology*, Department of Pharmacology, Physiology and Toxicology** and Preventive Medicine and Community Health Biostatistics Division ** University of Medecine and Dentistry of New Jersey Medical School, Newark, New Jersey 07103, and the Veterans Affair Medical Center*, East Orange, New Jersey 07019, Huashang Hospital, Shangai, People's Republic of China ${ }^{* * *}$.

Endocrinology, 138, 2, 553-559, 1997

Récemment, Huang et collaborateurs ont rapporté que l'administration d'une dose unique d'1 mg de testostérone à des rats âgés de 20 jours entraînait des modifications du taux de RNA messager des récepteurs de l'acide rétinoïque (RAR a et g) dans le testicule.

Cette observation démontre que le gène de RAR peut être modulé par les androgènes et suggère que le RAR peut être impliqué dans la régulation des fonctions sertoliennes par les androgènes. Les RAR sont importants comme médiateurs des actions biologiques de l'acide rétinoïque qui est essentiel pour la prolifération cellulaire, la différenciation et le développement.

C'est la raison pour laquelle nous nous sommes intéressés aux effets de la castration et de la testostérone sur l'expression du mRNA des RARs a et $\mathrm{g}$ dans la prostate, les vésicules séminales et le rein du rat adulte.

Il a été montré que, dans les conditions physiologiques normales, les taux des transcrits du mRNA de RARs a et $g$ dans la prostate du rat adulte, étaient au moins 20 fois supérieurs à ceux trouvés dans les vésicules séminales, le rein et la rate.

Bien que l'abondance du mRNA du RAR a dans la prostate soit comparable à celle du testicule, le taux du mRNA du RAR g a été trouvé 20 fois supérieur à celui du testicule.

Ces résultats suggèrent que les RARs a et $\mathrm{g}$ peuvent jouer des rôles importants dans la physiologie prostatique.

L'augmentation du taux de mRNA du RAR après castration et une diminution importante après administration de testostérone tant au niveau de la prostate que des vésicules séminales chez l'animal castré est en faveur d'une " down-regulation " des gènes RAR par la testostérone dans ces deux organes androgéno-dépendants. Il est donc suggéré que l'acide rétinoïque et/ou le RAR pourraient être impliqués dans l'action de la testostérone au niveau de la prostate et des vésicules séminales.

Il a été montré aussi une diminution des taux du mRNA des RARs a et $g$ dans le rein après castration et une récupération après administration de testostérone exogène. Ce phénomène $a$ donc une influence sur la concentration de très nombreuses protéines rénales : alcool deshydrogénase, ornithine décarboxylase, $\beta$-glucuronidase qui sont stimulées par les androgènes.

La vitamine A serait donc impliquée dans un très grand nombre de situations.

Au niveau testiculaire, les mRNAs du RAR diminuent au cours de la carence en vitamine $A$ et peuvent être stimulés par l'acide rétinoïque ou le rétinol. Au niveau des cellules de l'hépatome humain, on observe une stimulation des transcrits du mRNA pour le RAR b sous l'action de l'acide rétinoïque alors que ce n'est pas le cas pour le mRNA du RAR a.

Au niveau du carcinome embryonnaire F-9, on observe une stimulation des transcrits RAR $b$ du mRNA par l'acide rétinoïque alors que les formes a et $\mathrm{g}$ ne répondent pas à la vitamine.

L'implication de l'AMP cyclique a été suggérée dans la régulation de l'expression de RAR par les androgènes tant au niveau de la prostate que des vésicules séminales. L'administration de testostérone exogène à des rats castrés, semble stimuler l'AMP cyclique cellulaire qui, à son tour, 
entraîne une diminution des taux de mRNA du RAR dans la prostate et les vésicules séminales. Il apparaît donc que cette étude démontre la présence d'une quantité importante de transcrits de mRNA du RAR dans la prostate de rat adulte, dans les conditions physiologiques et que son expression peut être modulée par la testostérone.

Ces résultats, ainsi que les observations de métaplasie des cellules épithéliales prostatiques au cours de la carence en vitamine $A$, la prévention de la carcinogénèse prostatique par l'acide rétinoïque et ses analogues ainsi que l'inhibition par l'acide rétinoïque de la régulation par les androgènes de la prolifération cellulaire dans les vésicules séminales de la souris néonatale, soulignent l'importance de l'acide rétinoïque et de son RAR sur les effets des androgènes sur la physiopathologie prostatique.

\section{Commentaires (M. DROSDOWSKY) :}

La vitamine A intervient dans de nombreux processus biologiques :

1) action sur la croissance, en particulier chez le rat mais aussi chez l'homme adulte et surtout enfant (synthèses des protéoglycannes et des glycoprotéines, des hormones stéroüdes et des protéines ; stabilité des membranes)

2) rôle dans la vision

3) rôle dans le développement

4) enfin, rôle dans la reproduction, l'avitaminose A étant une cause de stérilité (modèles animaux)

La publication de Huang et al. montre que la vitamine $A$ non seulement intervient au niveau $d u$ tube séminifère du testicule, mais aussi au niveau des organes cibles de la testostérone comme la prostate et les vésicules séminales.
La cycline D2 est un gène répondant à la FSH et impliqué dans la prolifération cellulaire et l'oncogenèse gonadique

Cyclin D2 is an FSH responsive gene involved in gonadal cell proliferation and oncogenesis

P. Sicinski, J.L. Donaher, Y. Geng, S.B. Parker, H. GARDNER, M.Y. PARK, R.L. ROBKER, J.S.

RichaRds, L.K. MCGinNis, J.D. BigGERS, J.J. EPPIG, R.T. BRONSON, S.J. ElLEDGE, R.A. WEINBERG

Whitehead Institute for Biomedical Research, Cambridge, MA 02142 USA

Nature $1996 ; 384: 470-474$

Les cyclines de type D (D1, D2 et D3) sont des régulateurs fondamentaux de la chronologie du cycle cellulaire pendant la phase G1 chez les mammifères. Ces 3 cyclines s'expriment en même temps, de manière apparemment redondante, dans les tissus caractérisés par une prolifération cellulaire. Pour comprendre pourquoi les cellules avaient besoin de 3 cyclines D différentes, des souris transgéniques déficitaires pour le gène de la cycline $\mathrm{D} 2$ ont été générées par recombinaison homologue dans les cellules souches embryonnaires. Les souris homozygotes ont été obtenues par croisement des souris hétérozygotes avec une proportion en accord avec la transmission mendélienne. Bien que normalement l'expression de la cycline D2 soit largement répandue pendant le développement embryonnaire chez la souris, les souris Cyclin D2-/- ne présentaient pas d'anomalie et ne pouvaient être différenciées des souris sauvages, ce qui est bien en faveur de la redondance du système des cyclines $D$. Ce n'est qu'au moment de la reproduction qu'une différence est apparue. Les souris femelles déficitaires pour le gène de la cycline D2 étaient stériles en raison d'un déficit de prolifération des cellules de la granulosa en réponse à la FSH, aussi bien in vivo qu'in vitro, avec observation de follicules hypoplasiques contenant moins de cellules de la granulosa, multiplication minimale des cellules de la granulosa in vivo après injection de FSH et multiplication faible des cellules de la granulosa dans les follicules cultivés en présence de FSH. Par contre, la production d'estradiol était respectée. A la suite du traitement par la FSH, l'administration de LH ne permettait pas la 
libération de l'ovocyte par les follicules hypoplasiques qui étaient toutefois lutéinisés. Quand les ovocytes ont été recueillis manuellement, ils ont pu répondre à une maturation in vitro, être fécondés et donner lieu à un développement embryonnaire normal. Il apparaît ainsi que la cycline D2 est normalement nécessaire pour la prolifération des cellules de la granulosa. Ceci a été confirmé par le fait que les ARN messagers de la cycline D2, mais pas ceux des cyclines D1 et D3, sont augmentés par la FSH dans la granulosa des souris normales in vivo et in vitro. Cette action est médiée par l'activation de la protéine kinase A (la forskoline donne un effet identique) alors que les ARNm de la cycline D2 ne sont pas modifiés par l'activation de la protéine kinase $\mathrm{C}$.

Les mutants mâles ont des testicules identiques aux souris normales à la naissance. Toutefois, le volume testiculaire augmente 2,5 fois moins lors de la maturation sexuelle avec production de 2 à 2,9 fois moins de spermatozoïdes. La concentration de testostérone dans le sérum est normale. En dépit de leur taille inférieure à la normale, les testicules de souris D2-/- ont une structure normale en microscopie. Comme il a été montré que la cycline D2 s'exprime dans les cellules de Sertoli, on trouve une situation proche de celle de l'ovaire. Néanmoins, l'hypoplasie des testicules ne s'accompagne pas de stérilité complète.

L'hypoplasie des ovaires et des testicules observée chez les souris D2-/- a poussé à examiner les cancers dérivant de ces organes. Il a été trouvé que la plupart des tumeurs des cellules de la granulosa (10/12) contiennent des quantités élevées d'ARNm de la cycline $D 2$, contrairement aux autres types histologiques de tumeurs ovariennes. De même, plusieurs lignées cellulaires dérivées de tumeurs testiculaires à cellules germinales (carcinomes embryonnaires, tératocarcinomes) renferment des quantités élevées de cycline D2, avec augmentation du nombre de copies du gène de la cycline D2 dans 15 des 19 lignées cellulaires étudiées.

Ainsi il apparaît que la cycline D2, dont l'expression dans les gonades est stimulée par la FSH et dont l'invalidation provoque une réduction de la croissance cellulaire normalement induite par la FSH, se trouve exprimée de manière excessive dans des tumeurs dérivées des gonades.

\section{Commentaire (H. LEJEUNE)}

Les expériences d'invalidation génique par recombinaison homologue ne peuvent être, à l'heure actuelle, réalisées que chez la souris, seule espèce où les cellules embryonnaires totipotentes (cellules ES, pour Embryonic Stem cells) peuvent être isolées et peuvent se multiplier in vitro. Ceci permet leur transfection avec des constructions permettant le remplacement d'un gène par une copie modifiée, tout en gardant sa localisation normale dans le génome. Les expériences d'invalidation génique (knock out) apportent un certain nombre de surprises. Dans d'assez nombreux cas, l'invalidation d'un gène que l'on pense fondamental n'a que peu ou pas de conséquence. Ceci s'explique par la redondance des systèmes biologiques. C'est ce qui se passe pour la cycline D2. L'invalidation de son gène ne provoque pas d'anomalie du développement embryonnaire comme on aurait pu s'y attendre en raison de l'expression abondante de la cycline $D 2$ pendant la vie embryonnaire. Les autres cyclines $D$ ont très vraisemblablement remplacé la cycline $D 2$ manquante pour la plupart des effets. La présente étude montre, par contre, que dans les cellules cibles de la FSH, le phénomène de redondance n'est pas opérant. Les cyclines D1 et D3, sans doute parce qu'elles ne sont pas régulables par la FSH, ne parviennent pas à compenser l'invalidation de la cycline D2. L'effet de croissance cellulaire des cellules de la granulosa, normalement médié par la FSH qui augmente la production de cycline D2, ne se produit pas. Par contre, les autres fonctions ovariennes apparaissent respectées y compris la production d'estradiol qui met en jeu les cellules de la granulosa. Les résultats présentés chez les mâles sont plus parcellaires, sans doute en raison de l'effet moins net obtenu, avec certes, diminution de la taille du testicule et diminution de la production de spermatozoïdes, mais absence de stérilité complète. Ceci est toutefois en bon accord avec ce que l'on sait de l'effet de la FSH sur la croissance des cellules de Sertoli. Si la croissance des cellules de la granulosa, sous l'effet de la FSH, se produit à chaque cycle, la croissance des cellules de Sertoli n'a lieu que lors de la maturation testiculaire. L'effet de la FSH chez le mâle ne porte ensuite plus tellement sur la multiplication cellulaire, mais plus sur la synthèse protéique des cellules de Sertoli. L'importance de la FSH chez le mâle adulte est même discutée pour les rongeurs. L'augmentation de l'expression de la cycline D2 dans les tumeurs de 
la granulosa apparaît un fait concordant avec le rôle de la cycline D2 dans la croissance cellulaire de ces cellules, tout au moins dans la composante induite par la FSH. Savoir dans quelle mesure cette hyper-expression de la cycline D2 est un phénomène initial dans la carcinogenèse des cellules de la granulosa nécessitera des travaux complémentaires. En ce qui concerne le testicule, les investigations présentées portent sur des lignées cellulaires dérivées de tumeurs à cellules germinales et non pas de tumeurs à cellules de Sertoli. Le lien avec le rôle de la cycline D2 dans la médiation des effets de la FSH sur la multiplication cellulaire est donc moins clair.

\section{ASSISTANCE MEDICALE A LA PROCREATION}

Index de difformité spermatique : prédication fiable de la fécondation in vitro

The sperm deformity index: a reliable predictor of the outcome of oocyte fertilization in vitro

Aziz N, Buchan I., Taylor C., Kingsland Ch., LEWIS-JONES I

Fertil-Steril, 1996, 66, 1000-1008

\section{Résumé}

Afin de permettre une meilleure approximation des chances de réussite d'une FIV, l'étude des anomalies morphologiques du sperme et la capacité de celui-ci à prédire la fécondation d'un ovocyte seront déterminées par la comparaison de trois indicateurs :

- le pourcentage des formes normales, basé sur les critères stricts de normalité morphologique,

- l'index de difformité spermatique qui évalue le nombre total d'anomalies morphologiques observées par le nombre de spermatozoïdes séléctionnés au hasard indépendamment de leur normalité morphologique, et

- l'index d'anomalies multiples (IAM) qui se calcule en divisant le nombre d'anomalies constatées par le nombre de spermatozoïdes anormaux. L'intérêt de ce nouvel index est d'attribuer à un spermatozoïde un index d'autant plus élevé que le nombre d'anomalies morphologiques constaté est important sans favoriser telle anomalie sur telle autre. L'IDS par rapport au \% des formes normales apparaît comme un prédicteur fiable pour la sélection du sperme des patients inclus dans un programme d'ICSI.

\section{Résultats}

Cette étude a été réalisée sur 158 couples. Les ovocytes à trois pronuclei ont été comptabilisés dans le nombre d'ovocytes fécondés mais n'ont pas été considérés dans le nombre d'embryons normalement divisés. Les spermes ont été analy- 
sés selon l'OMS, et la morphologie des spermatozoïdes selon les critères stricts de normalité morphologique (coloration au Spermac). L'IDS est calculé à partir d'une méthode proche de celle d'Eliasson: cette classification morphologique stricte nécessite une pratique régulière. L’index de difformité spermatique (IDS) a été calculé en divisant le nombre total d'anomalies morphologiques du spermatozoïde par le nombre de spermatozoïdes sélectionnés au hasard indépendamment de leur normalité morphologique, de même pour l'index d'anomalies multiples mais en ne tenant compte que des spermatozoïdes anormaux. Le pourcentage de spermatozoïdes normaux a été évalué pour tous les échantillons étudiés.

Sur 158 couples : $3 / 4(116 / 158)$ ont eu une fécondation avec un taux compris entre 10 et $100 \%$. Parmi ces 158, 134 patients ont un sperme normal selon la numération et la mobilité, 24 sont soit oligo et/ou asthénospermiques avec un $\%$ des formes normales variant entre 1 et $50 \%$. Parmi les 42 couples qui n'ont pas eu de fécondation, 23 ont un sperme normal, 6 sont oligospermiques, 10 asthénospermiques, 3 oligoasthénospermiques avec un \% des formes normales variant entre 0 et $17 \%$.

Le calcul de l'IDS a permis de déterminer une valeur seuil grâce à la courbe de ROC fixée à 1,6. IDS $\leq 1,6$ a une valeur prédictive positive de $90 \%$ (111 réussites prédites $/ 116$ cas) versus IDS $\geq 1,6$ a une valeur prédictive négative de $86 \%$ (30 échec prédits / 42 cas).

La comparaison entre l'IDS et le \% des formes normales $(4 \%)$ dans cette étude, montre que l'IDS possède une plus grande spécificité et sensibilité respectivement $(71 \%, 96 \%)$ que le $\%$ de FN $(69 \%, 87 \%)$ et donc permet une meilleure approximation des chances de réussites de la fécondation. En comparant le cut-off de l'IAM $(1,75)$ et l'IDS $(1,60)$, il apparaît que l'IAM est moins spécifique mais aussi sensible que l'IDS. Il se révèle être un bon test négatif avec des prévisions similaires à l'IDS.

\section{Commentaires (S. HAMAMAH)}

Des critiques peuvent être faites à l'encontre de cette étude, en particulier la trop grande disparité quant à l'âge de la femme, la durée de l'infécondité, et surtout le nombre d'ovocytes mis en culture (2 à 23) pour le groupe ayant eu une fécondation en FIV et (2 à 13) pour le groupe sans fécondation, rendant l'interprétation des résultats de cet article très diffi- cile. De plus, l'IDS ne semble pas influencer la survenue d'une grossesse évolutive dans la mesure où l'IDS n'est pas significativement différent entre le groupe des couples ayant eu des embryons sans aboutir à une grossesse par rapport aux couples ayant eu une grossesse menée à son terme.

La méthode par laquelle l'IDS a été établie est peu claire (nombre de spermatozoïdes étudiés non précisé). La différence avec l'index d'anomalies multiples ne ressort pas nettement (quelles difformités peuvent être trouvées sur des spermatozoïdes dits normaux ?). Comme l'IDS a été réalisé à partir d'une méthode proche de celle d'Eliasson nécessitant une classification morphologique stricte, le calcul de lIDS a besoin d'une pratique régulière. L'utilisation de l'IDS me semble illusoire à l'heure actuelle .

\section{Modalités de récupération de la fertilité après vasectomie : analyse du coût et de l'efficacité}

Fertility options after vasectomy : a cost-effectiveness analysis

\author{
C.P. Pavlovich*, P.N. Schlegel** \\ * The New York Hospital-Cornell Medical Center \\ ** The Population Council, New-York \\ Fertility and Sterility, $67, \mathrm{n}^{\circ} 1,133-141$
}

Aux Etats Unis $13 \%$ des couples mariés ont recours à la vasectomie comme méthode de contrôle des naissances. $2 \%$ à $6 \%$ des hommes demandent une réanastomose pour restaurer leur fertilité. Les forts taux de grossesses obtenus par ICSI après ponction de spermatozoïdes ont fait s'interroger sur la nécessité de la réanastomose. Les taux de grossesses obtenues après ICSI varient en effet, de $18 \%$ à $41 \%$ par tentative suivant les centres.

Nous avons cherché à évaluer les deux approches en termes de taux d'accouchements et de coût global par accouchement.

\section{Matériel et Méthodes}

Les données concernant l'ICSI ont été collectées dans 4 centres ( 710 cycles), les données concernant les réanastomoses (vasoépididymostomies, vasovasostomies) proviennent de 5 grands centres et d'une étude multicentrique. 


\begin{tabular}{lcr}
\hline ICSI après ponction de sperme & $27,7 \%$ par tentative & $72.521 \$$ \\
\hline Réanastomose & & \\
suivant le délai après la vasectomie & $68 \%$ & $20.594 \$$ \\
$<3$ ans & $48 \%$ & $25.072 \$$ \\
de 3 à 8 ans & $40 \%$ & $28.118 \$$ \\
de 9 à 14 ans & $27 \%$ & $36.915 \$$
\end{tabular}

\section{Discussion}

Il apparaît clairement que le coût global par accouchement est nettement moindre et le taux d'accouchements nettement meilleur dans la réanastomose que dans l'ICSI après ponction de spermatozoïdes. Les grossesses multiples liées à l'ICSI accroissent considérablement le coût.

Nous suggérons de recueillir et cryoconserver des spermatozoïdes obtenus par ponction au cours de l'intervention chirurgicale pour réanastomose afin d'éviter les multiples ponctions en cas de recours ultérieur à l'ICSI.

\section{Commentaires (J.C.CZYBA)}

Les auteurs ont procédé à l'étude de nombreux paramètres et utilisé des méthodes statistiques raffinées dont notre résumé ne tient pas compte.

Ils prétendent apporter les éléments qui permettent, en évaluant les coûts, bénéfices et risques, la prise de la décision thérapeutique. Il s'agit non seulement d'informer les patients mais aussi "tout tiers payant intervenant dans la prise en charge du traitement de la stérilité».

Nous avons, pour notre part, été très étonné (après deux lectures attentives de l'article) de ne trouver aucune proposition de recours à la cryoconservation du sperme avant la vasectomie. Cette pratique, largement répandue en France, est justifiée par le fait que le spermogramme est en principe de bonne qualité avant l'intervention et que la pratique ultérieure, éventuelle, d'inséminations artificielles donne de bons résulats (environ 70\% d'accouchements pour une moyenne de 3 cycles et un coût de quelques milliers de francs, c'est-à-dire quelques centaines de dollars). La méthode n'exclut pas le recours préalable à l'anastomose si le couple répugne à recourir aux IAC en première intention et préfère une grossesse résultant directement d'un rapport sexuel ; elle permet, en tout cas, si besoin est, le recours à la FIV et à l'ICSI sans ponction de spermatozoïdes. 